\title{
Small Animal Radiation Research Platform: Imaging, Mechanics, Control and Calibration
}

\author{
Mohammad Matinfar ${ }^{1}$, Owen Gray ${ }^{1,2}$, Iulian Iordachita ${ }^{1}$, Chris Kennedy ${ }^{2}$, \\ Eric Ford ${ }^{2}$, John Wong ${ }^{2}$, Russell H. Taylor ${ }^{1}$, and Peter Kazanzides ${ }^{1}$ \\ ${ }^{1}$ Dept. of Computer Science, Johns Hopkins University, Baltimore, MD \\ 2 Dept. of Radiation Oncology, Johns Hopkins Medical Institution, Baltimore, MD *
}

\begin{abstract}
In cancer research, well characterized small animal models of human cancer, such as transgenic mice, have greatly accelerated the pace of development of cancer treatments. The goal of the Small Animal Radiation Research Platform (SARRP) is to make those same models available for the development and evaluation of novel radiation therapies. In combination with advanced imaging methods, small animal research allows detailed study of biological processes, disease progression, and response to therapy, with the potential to provide a natural bridge to the clinical environment. The SARRP will realistically model human radiation treatment methods in standard animal models. In this paper, we describe the mechanical and control structure of the system. This system requires accurate calibration of the x-ray beam for both imaging and radiation treatment, which is presented in detail in the paper.
\end{abstract}

\section{Introduction}

The tremendous advances in medical imaging technologies over the last decade are revolutionizing the management of patient treatment and care. In cancer therapy, high speed, high resolution anatomical imaging 112 in combination with functional imaging 34 significantly improve our ability to stage the disease, localize the tumor, and evaluate the treatment process. It has also become apparent that by applying advanced imaging methods to study small animals, such as mice or rats, much can be gained in our understanding of the disease processes and the development of new treatment strategies. Micro-animal systems capable of very high-resolution have been developed in positron emission tomography [56], X-ray CT [78, magnetic resonance imaging 9910, magnetic resonance spectroscopic imaging [11] and ultrasound imaging [12].

Modern image guided conformal beam radiotherapy aims to deliver a high therapeutic dose to cancerous tissue while minimizing the dose to healthy tissue. Delivering the therapeutic dose from multiple poses during a single session allows a concentrated dose to be delivered to the tumor, while reducing the dose to surrounding tissue. To do this effectively, the location of the tumor must be known, requiring pretreatment scans and registration of the treatment beam.

^ This work was supported by NIH 1 RO1 CA108449-01.

N. Ayache, S. Ourselin, A. Maeder (Eds.): MICCAI 2007, Part II, LNCS 4792, pp. 926 9342007.

(C) Springer-Verlag Berlin Heidelberg 2007 
Because there is no safe dose of radiation, it is critical that the risks of radiation exposure are balanced against the efficacy of the treatment.

Given the size, complexity, and expense of imaging equipment and therapeutic linear accelerators, most novel techniques and protocols are first tested on human subjects. This poses a number of technical and ethical dilemmas because there is only limited pre-clinical validation of most techniques and the number of subjects in clinical trials is typically small, with participants having some of the most dire prognoses after standard techniques have failed. The SARRP aims to address these issues by providing a platform that can perform high-resolution imaging and accurate conformal beam therapy on standard animal models for human cancers. Currently, radiotherapy trials on animal models use gamma cells or similar devices. The SARRP offers several advantages over the gamma cell, including portability and the ability to deliver radiation over a conformal arc rather than as single beams. Many mouse models of human cancer are currently available, but existing imaging and therapeutic systems are ill suited to such small subjects, and the equipment is also in high demand and seldom available for lengthy laboratory trials. The SARRP provides cone beam CT imaging, radiation therapy, and correlates this with treatment dose and efficacy.

We believe that this is the first system being developed for the purpose of radiation therapy research on small animals. Section 2 discusses the mechanics and control structure. Sections 3 and 4 describe the imaging and treatment subsystems, respectively, with a focus on calibration methods.

\section{Mechanics and Control Structure}

SARRP integrates imaging, radiation delivery and treatment planning capabilities. The mechanical structure is designed to meet the system requirements, which are to attain CT voxel resolution of $0.5 \mathrm{~mm}$ or better with 1cGy imaging dose, localized radiation dose at a FWHM of $1.5 \mathrm{~mm}$, and a dose rate of 200 cGy per minute. The following sections describe the major components:

Robotic Positioner: The robotic positioner $\theta \mathrm{XYZ}$ consists of three modular offthe-shelf subassemblies: rotating table, X-Y cross table, and vertical stage, Fig.1. The rotary table is a preloaded, anti-backlash worm assembly that provides exceptional angular accuracy (0.05 degrees) and repeatability (0.007 degrees). It is actuated by an encoded DC motor. This stage provides unlimited angular positioning but, due to the cabling to the the XYZ axes, the range of motion is limited to $\pm 190 \mathrm{deg}$ via software. The $\mathrm{X}-\mathrm{Y}$ motions are realized by an $\mathrm{X}-\mathrm{Y}$ cross table. Each axis consists of a ball way table actuated by an encoded DC motor driven lead-screw (65 $\mu \mathrm{m}$ /axis accuracy), with a monolithic center. The unit incorporates anti-backlash friction nuts to achieve a high repeatability (6 $\mu \mathrm{m})$. The motion range for each axis is $\pm 50 \mathrm{~mm}$. For the vertical (Z) motion, we employed a stage with a scissors mechanism, motorized with an encoded DC motor. The travel length is $38 \mathrm{~mm}$ and the anti-backlash lead-screw assembly has good repeatability $(0.125 \mathrm{~mm})$. Although less precise than the other stages, it is sufficient to satisfy the design requirements. 


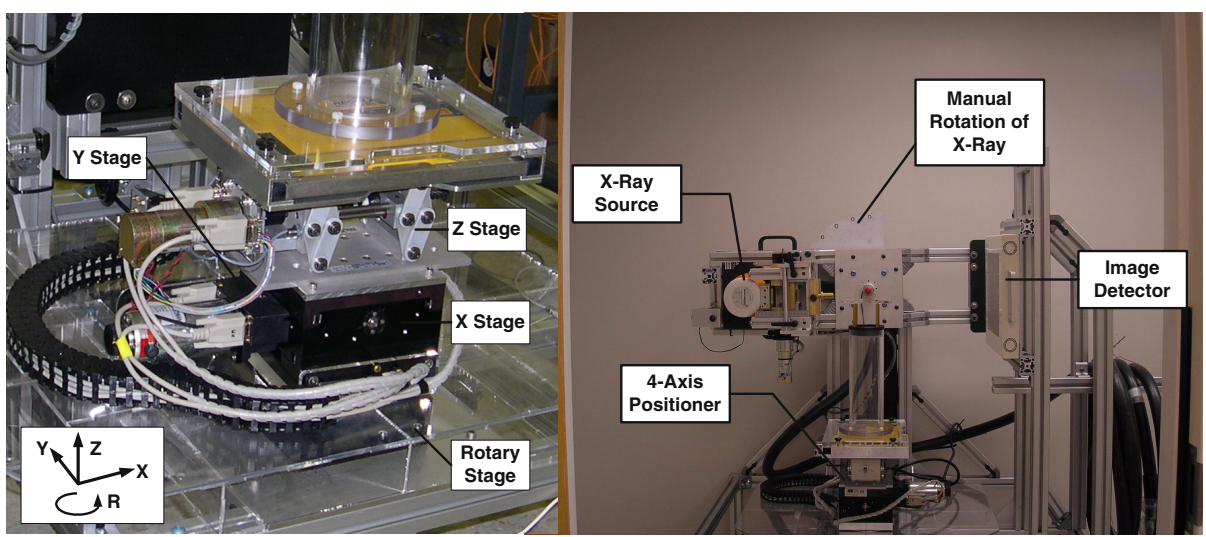

Fig. 1. Different components of the Small Animal Radiation Research Platform

X-Ray Tube and Arm: The X-ray source has a variable output with a maximum beam energy of $225 \mathrm{kV}$ p. Images are acquired using $100 \mathrm{kVp}$ photons and a spot size of $0.2 \mathrm{~mm}$. In treatment mode, $225 \mathrm{kVp}$ photons are generated from a $2 \mathrm{~mm}$ focal spot with up to $13 \mathrm{~mA}$ beam current to deliver clinically useful dose rates ( $\sim 2 \mathrm{~Gy} / \mathrm{Min})$ down to $0.5 \mathrm{~mm}$ FWHM beam. The X-ray source is mounted on a rotary arm which can be manually set at nine different positions. These positions are located 15 degrees apart to create a 120 degree motion range. Any location of the arm can be used for radiation therapy, whereas cone beam imaging is only possible when the source is in the horizontal position.

Collimator and Shutter: There are three collimators aligned with the x-ray beam axis. The primary collimator, fixed to the x-ray source, reduces the size of the beam to $200 \times 200 \mathrm{~mm}$ at the isocenter and is permanently attached. The secondary collimator has a fixed position, but can be easily removed; it reduces the beam size to $60 \times 60 \mathrm{~mm}$ at the isocenter. The third collimator has a variable position along the x-ray axis ( $\min 230 \mathrm{~mm}$, $\max 310 \mathrm{~mm}$ ), is easily removable (together with the second one), and can be chosen to set the beam diameter as low as $0.5 \mathrm{~mm}$. The shutter is a motor driven linear stage carrying two brass pieces to block the x-ray radiation during the tube power-up phase.

Image Detector Panel: Image acquisition is accomplished with a flat panel digital x-ray detector. The current system is a $512 \times 512$ pixel array with $0.4 \mathrm{~mm}^{2}$ pixel size and 16 bit resolution. The detector frame rate is $7 \mathrm{~Hz}$, providing rapid acquisition of images for cone beam tomography. Preprocessing of acquired images is performed in hardware on the frame grabber card to maximize the acquisition rate and allow concurrent reconstruction of $\mathrm{CT}$ volumes on a standard PC workstation. Dark current and gain correction images are acquired prior to each imaging run and used to correct pixel intensities as each image is acquired. 
Control Structure: The control structure of the SARRP has two main components: a PC with a graphical user interface connected, via Ethernet, to an intelligent motion control board. The motion control board contains a 32-bit microprocessor that provides PID control of up to 6 motors, with a loop update rate of 500 microseconds.

Laser Alignment System: The SARRP provides a laser alignment system to facilitate accurate, reproducible setup of subjects. A removable line laser is mounted on the x-ray tube and a cross-hair laser permanently mounted on the gantry pivot. These lasers converge at the nominal isocenter and enable visual setup of large treatment fields, where an accuracy of several millimeters is sufficient. Frequently, tumors are implanted in the extremities (flank or dorsal fold) of subjects, and the laser alignment provides a rapid means of assessing setup accuracy without the need to acquire x-ray images.

Animal Support Fixture: While the laser system is intended for relatively coarse

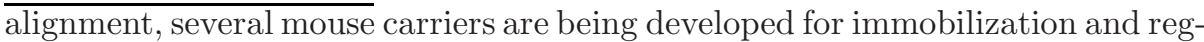
istration, where millimeter or submillimeter accuracy is required. These mouse carriers incorporate gas anesthesia, temperature control to prevent hypothermia, and stereotactic frames for accurate delivery of treatment beams to the cranium. The devices are MR compatible, and provide fiducials for coregistration of PET, $\mathrm{MR}$, and CBCT volumes.

Moreover, the SARRP provides integrated portal imaging using a standard $\mathrm{x}$-ray film cassette mounted below the subject in the anterior-posterior plane. The portal image may be used to confirm setup accuracy post treatment as well as to perform the initial setup.

\section{Imaging Subsystem}

The imaging system uses a novel geometry to acquire cone beam CT sequences. The animal rests on a rotating platform and is rotated around an anteriorposterior axis. Images are acquired with the x-ray source in the horizontal position. As the subject is rotated, a series of projection radiographs are acquired. Volumetric reconstruction is accomplished using filtered back projection [13]. This geometry poses a problem due to the large disparity in path lengths when the beams traverse the long axis of the subjects relative to the lateral axis. Due to the additional attenuation, ringing and "cupping like" artifacts are present in the reconstructed volume. It is believed that this is due to beam hardening, and additional simulation studies are planned to develop a correction scheme to provide accurate density information for treatment planning and dose calculation.

The SARRP imaging beam operates at $100 \mathrm{kVp}$ with $0.5 \mathrm{~mm} \mathrm{CU}$ and $2 \mathrm{~mm}$ AL filtration. The pre-hardening of the beam is necessary to reduce artifacts from beam hardening. The aim of the imaging subsystem is not high resolution $\mathrm{CT}$, but rapid, low dose acquisition and quantitative CT for dose calculation and treatment planning. Existing micro CT scanners use relatively low beam energies on the order of $20 \mathrm{kVp}$. While this provides excellent contrast and resolution, the 


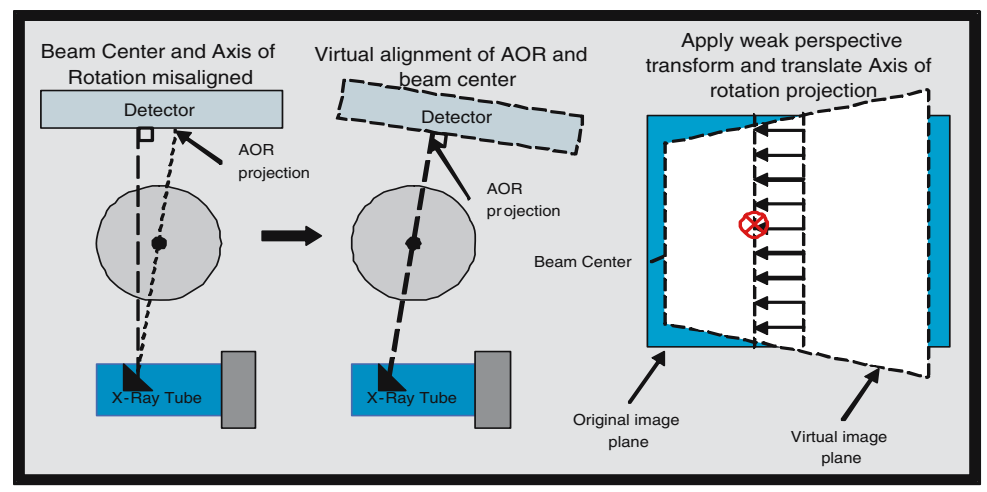

Fig. 2. Imaging subsystem of the SARRP

imaging dose to the subject is high relative to the SARRP system. The SARRP imaging dose is roughly $10 \mathrm{cGy}$, which is an order of magnitude lower than a typical therapeutic dose. High imaging doses confound any analysis of subsequent radiotherapy, and in many cases are sufficient to induce immunosuppression.

\subsection{Camera Calibration}

Camera calibration is accomplished using 2D-3D point correspondences in the method described by Bopp [14. A fiducial object composed of point-like features (BBs or holes in a thin copper plate) with known geometry is imaged, and 6 or more landmarks are used to calculate the intrinsic (focal length and detector offset) and extrinsic (detector position in world coordinates) camera parameters.

Input images are thresholded, connected components are extracted, and the center of mass is calculated. The user then manually inputs the known positions of each object in world coordinates ( $\mathrm{Z}$ axis is vertical, $\mathrm{X}$ axis is from source to detector, and $\mathrm{Y}$ axis is transverse).

\subsection{Geometric Calibration}

For accurate cone beam reconstruction using the Feldkamp algorithm [13, it is essential that the axis of rotation of the subject intersect the beam axis (the ray normal to the detector surface passing through the x-ray source). The axis of rotation of the theta stage is calculated by rotating a fiducial object composed of point-like objects, Fig. 2. Using the known camera geometry, the axis of rotation in world coordinates and its projection on the detector may be calculated. In practice, perfect alignment of the system is not possible, so additional correction may be applied to the projection radiographs. To correct any residual misalignment, a weak perspective transform is applied to the projection images to create a virtual alignment of the axis of rotation and beam axis. The projection of the axis of rotation is shifted, and an intensity correction is applied to all pixels to account for the inverse square drop off in intensity for the aligned image. 


\section{Treatment (Therapy) Subsystem}

Radiation therapy is often delivered from multiple poses that are intended to intersect at a specified point (the treatment isocenter). Positioning the target (tumor) at this point will cause it to receive a higher radiation dose than the surrounding healthy tissue. With the SARRP, rotation about a target can be obtained via the rotary (theta) axis or the x-ray arm. At a given x-ray arm orientation, 1 the isocenter can be defined by the closest intersection of the two $3 \mathrm{D}$ lines defined by the $\mathrm{x}$-ray beam and the rotary axis center of rotation (in general, two 3D lines will not intersect, but a closest intersection can be defined on the shortest line that is perpendicular to both). Because the x-ray arm is manually positioned, it is feasible to define nine different isocenters - one for each position. Figure 4 (right) illustrates the concept, where only 5 treatment positions ( $\mathrm{P} 0-\mathrm{P} 4)$ and 2 isocenters $(\mathrm{C} 1, \mathrm{C} 4)$ are shown for visual clarity. If the offsets between the nine isocenters are measured, the system can compensate by moving the XYZ axes whenever the x-ray arm is moved. This ensures that the target receives the maximum dose regardless of the position of the x-ray arm. If the beam axis and rotation (theta) axis do not exactly intersect, and the treatment plan requires rotation of the theta axis, it is possible to further compensate via coordinated motion of the XY axes with the theta axis. The following sections describe the techniques that were used to measure, and correct, the misalignments and offsets between the different axes.

\subsection{Offline Calibration}

For offline calibration, we used an optical tracking system to collect points on different components of the SARRP, as shown in Fig. 3. The collected data were analyzed to determine parameters such as the rotary stage and x-ray arm centers of rotation. Because the optical tracking system cannot sense the x-ray beam, all measurements were performed with the beam off. We estimated the beam axis by digitizing the x-ray opening window frame and taking into account the mechanical dimensions of the tube (e.g., from the manufacturer drawings). We performed three mechanical adjustments to improve the alignment. After these adjustments, the shortest distance between rotary stage center of rotation and the x-ray arm center of rotation was reduced to $0.30 \mathrm{~mm}$. The shortest distance between the estimated beam axis and the rotary stage axis of rotation was reduced to $0.31 \mathrm{~mm}$.

\subsection{Online Calibration}

Offline calibration is useful during initial construction and testing, but is not practical for online (periodic) use due to its requirement for a large and expensive optical tracking system. In contrast, the online calibration setup consists of a collimated beam (e.g., 1mm) and an x-ray camera, as shown in Fig. 囵(left). This

${ }^{1}$ Except when the x-ray arm is in the vertical position; in this case, the isocenter is defined at a specified distance along the beam axis. 

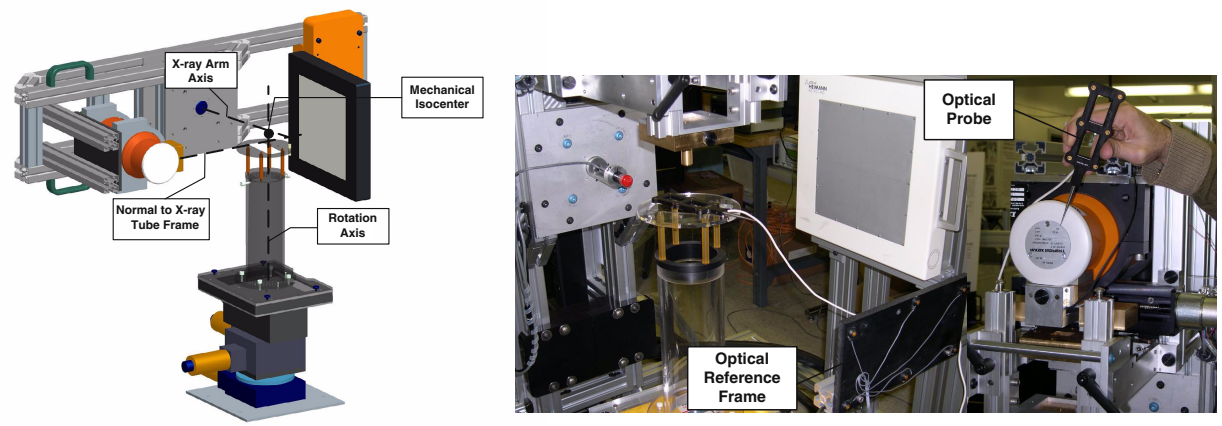

Fig. 3. Mechanical isocenter and data collection on different components of SARRP

method does not require precise positioning or calibration of the x-ray camera. As a first step, we measure the axis of rotation of the robotic positioner. This is accomplished by placing the x-ray arm in the vertical position and rotating the stage through a set of angles. The x-ray camera captures an image at each angle; these images are superimposed (added), as shown in Fig. 4 (center). The center of rotation, $C_{r}$ (in camera coordinates), is given by the center of gravity of the final image. Once $C_{r}$ is determined, we measure the isocenter at each x-ray arm position as follows:

1. Capture image with x-ray camera and compute center of gravity, $C_{i}$.

2. Move positioner (in XYZ) and repeat Step 1 until $C_{i}$ equals $C_{r}$. The amount of XYZ motion provides the coordinates of one point on the beam axis.

3. Move positioner along nominal beam axis by a "reasonable" amount (e.g., $10 \mathrm{~mm}$ ) and repeat Step 2. This provides a second point on the beam axis.

4. Isocenter is given by intersection of beam axis and axis of rotation.

The above method provides the relative position (offset) between the treatment isocenters in the positioner coordinate system. For convenience, we define the origin when the x-ray arm is in the horizontal (imaging) position. If the target is defined in the image coordinate system (e.g., subsequent to a cone beam CT scan), then the location of the isocenter in image coordinates is known (from the image calibration) and an isocenter offset is applied when the treatment beam is not in the imaging position. If lasers are used to align the target (e.g., with skin markers), then it is necessary to determine the offset between the laser isocenter and the beam isocenter.

\section{Conclusion and Future Work}

In this paper, we presented a novel system to integrate imaging, radiation delivery, and treatment planning for small animal research. The system was designed and constructed for development and evaluation of novel radiation therapies. The mechanical and control structure of the system were described and the calibration methods for x-ray beam imaging and radiation treatment were presented. 

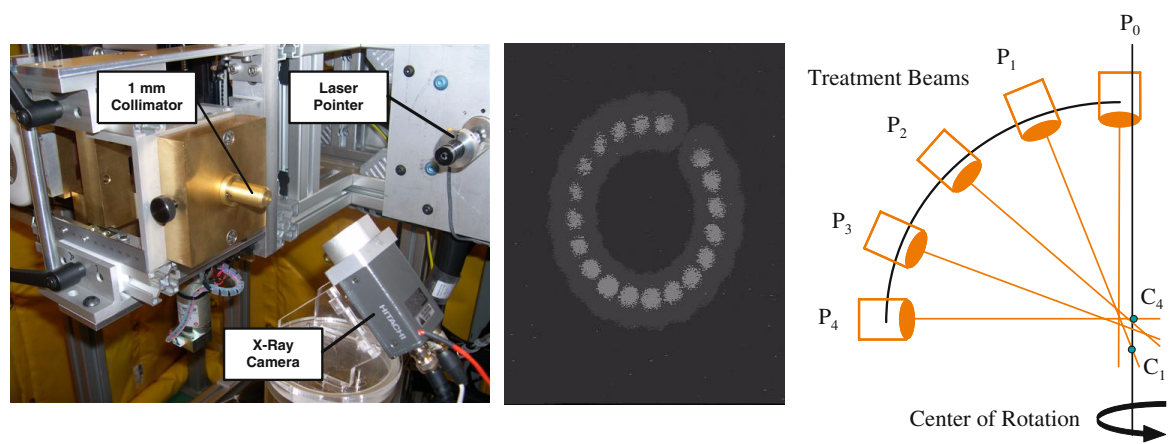

Fig. 4. Calibration setup, x-ray image used to find axis of rotation, and isocenter definition for SARRP

Future work will include validation of the calibration method, with central focus on determining the absolute accuracy of beam delivery. This will include characterizing and correcting for errors in the imaging system, beam alignment, and subject setup. The first phase will be to determine the accuracy with which a lesion observed in a reconstructed CT volume can be targeted, given the known error of the various subsystems. This will be followed by phantom studies to validate the accuracy of beam targeting.

\section{References}

1. Leter, E., et al.: Definition of a moving gross target volume for steretactic radiation therapy of stented coronary atretries. Jour. of Rad. Oncol. Biol. Phys. 52 (2002)

2. Schoef, U., et al.: Multi-slice computed tomography as a screening tool for colon cancer, lung cancer and coronary artery disease. Jour. of Eur. Rad. 11 (2001)

3. DiBiase, S., et al.: Magnetic resonance spectroscopic imaging-guided brachytherapy for localized prostate cancer. Jour. of Rad. Oncol. Biol. Phys. 52 (2002)

4. Nelson, S.: Analysis of MRI and MR spectroscopic imaging data for the evaluation of patients with brain tumors. Jour. of Mag. Res. Imag. 46 (2001)

5. Jacob, R., Cherry, S.: Compelementry emerging techniques: high-resolution PET and MRI. Current Opinion in Neurobiology 11 (2001)

6. Rubins, D., et al.: Evaluation of a stereotactic frame for repositioning of the rat brain inserial PET imaging studies. Jour. Neuroscience Methods 107 (2001)

7. Medynsky, A., et al.: Elastic response of numan iliac arteries in-vitro to balloon angioplasty using high-resolution CT. Jour. of Biomech 31 (1998)

8. Wan, S., et al.: Multi-generational analysis and visualization of the vascular tree in 3D micro-CT images. Comput. Biol. Med. 32 (2002)

9. Allport, J., Weissleder, R.: In vivo imaging of gene and cell therapies. Exp. Hematol. 29 (2001)

10. Franconi, F., et al.: In vivo quantitative microimaging of rat spinal cord at $7 \mathrm{~T}$. Jour. of Mag. Res. Imag. 44 (2000) 
11. Zhang, X., Ugurbil, K., Chen, W.: Microstrip RF surface coil design for extremely high-field MRI and spectroscopy. Jour. of Mag. Res. Imag. 46 (2001)

12. Turnbull, D., et al.: Ultrasound backscatter microscope analysis of mouse melanoma progression. Ultra. Med. Biol. 22 (1996)

13. Feldkamp, L.A., Kress, L.D.: Practical cone-beam algorithm. Optical Society of America A(1), 612-619 (1984)

14. Bopp, H., Krauss, H.: An orientation and calibration method for non-topographic applications. Optical Engineering 44(9), 1191-1196 (1999) 